\title{
Emerging micro- and nanotechnologies in cancer diagnosis and therapy
}

\author{
John X. J. Zhang • Ali Khademhosseini
}

Published online: 29 June 2013

(C) Springer Science+Business Media New York 2013

Understanding the mechanism of cancer cell metastasis is crucial for targeted cancer diagnosis and research. But this process is still not fully understood mainly because of lack of in vitro cell culture models that precisely mimic the physiological complexities of cancer cell intravascular flows, invasion, adhesion, metastasis, angiogenesis and migration to specific sites. Micro- and nanoscale technologies offer a promising tool for such purpose.

This special issue broadly covers the topics of cell microenvironment, biological surface modifications, multifunctional nanoparticles and nanosensors, microfluidics, molecular detection as well as multistage targeting strategies for cancer. In particular, it focuses on investigating how we can harness the power of micro- and nanotechnologies to radically change the way we diagnose and treat cancer. This includes both in vivo and ex vivo technologies. For in vivo applications, biosensors would have the capability of detecting tumors and metastatic lesions that are far smaller than those detectable using conventional technologies. Novel and multi-functional nanodevices will be capable of detecting cancer at its earliest stages, pinpointing its location within the body, delivering anticancer drugs specifically to malignant cells, and determining

\section{J. Zhang $(\bowtie)$}

Department of Biomedical Engineering,

The University of Texas at Austin, Austin, TX, USA

e-mail: john.zhang@engr.utexas.edu

A. Khademhosseini

Center for Biomedical Engineering, Department of Medicine, Brigham and Women's Hospital, Harvard Medical School, Cambridge, MA 02139, USA

A. Khademhosseini

Harvard-MIT Division of Health Sciences and Technology,

Massachusetts Institute of Technology, Cambridge, MA 02139, USA

\footnotetext{
A. Khademhosseini

Wyss Institute for Biologically Inspired Engineering, Harvard University, Boston, MA 02115, USA
}

if these drugs are effective. In addition, functionalized nanoparticles would deliver multiple therapeutic agents to tumor sites in order to simultaneously attack multiple points in the pathways involved in cancer. Such nano-therapeutics are expected to increase the efficacy of drugs while dramatically reducing potential side effects. For ex vivo application, the tumor microenvironment and circulating tumor cells can be studied. Together, such innovative approaches can provide information on potential early diagnosis as well as whether a given therapy is working as expected.

The group of Fischbach et al. reviews recent efforts in modulating the tumor microenvironment to develop in vitro tumor models for cancer pathogenesis and drug discovery. The work covers some of the recent landmark efforts towards this direction or micro-tumor engineering with focus on tissue microstructure organization and surrounding mass transport behavior using biomimetic approaches. The authors thus highlight the immense potential of microfabrication techniques for such cancer pathogenesis applications. In order to understand the recent advances in microfluidic technologies for cancer research, Nagrath et al. have identified four critical areas of research: cancer cell isolation, molecular diagnostics, tumor biology, and high-throughput screening for therapeutics. Cancer cell isolation can be further subcategorized into two major techniques: immunoaffinity-based isolation and size-based separation. Other methods include hydrodynamic focusing and dielectrophoresis. For the cancer cell isolation technologies, Ohnaga et al. developed polymeric microfluidic devices for the isolation of circulating tumor cells using microposts coated with anti-epithelial cell adhesion olecule antibody, and a flow system to capture tumor cells using an esophageal cancer cell line, KYSE220, dispersed in phosphate-buffered saline or mononuclear cell separation from whole blood. Recently, He et al. developed biocompatible nano-films composed of $\mathrm{TiO}_{2}$ nanoparticles to enhance topographic interactions between the nanofilm substrate surface and cell surface. The surface of the $\mathrm{TiO}_{2}$ nanoparticles is chemically modified to enable functionalization of anti-EpCAM antibodies. The 
design and implementation of the $\mathrm{TiO}_{2}$ nanoparticles serves to perform enhanced immunoassay capture of cancer cells.

It was also noted that CellSearch is the only FDA approved device for isolation of circulating tumor cells (CTCs) in metastatic breast, colon, and prostate cancers. The CellSearch system utilizes EpCAM-coated magnetic beads and correlation between numbers of isolated CTCs to provide prognosis in breast cancer patients. Molecular diagnostics can be used to monitor biomolecules such as circulating tumor DNA, microRNAs, proteins, and serum microvesicles. Microfluidic devices have the capability to detect such biomarkers with high sensitivity and accuracy. Conventional methods, such as digital/RT-PCR (TaqMan) and mi-RNAprofiling (Geniom Biochip), when miniaturized, enable high throughput capabilities and parallelization. Microfluidics can also aid in understanding tumor biology by mimicking the physiological cues in the cellular environment through spatial and temporal control over gradients of soluble factors and cell-cell contacts in the extracellular matrix. In addition, the cell culturing module can be integrated with downstream analysis such as imaging and molecular characterization. Finally, microfluidics has the unique advantages that allow highthroughput biomarker and drug screening.

Many groups are working towards integration of multiple components on a single chip to allow for culture, capture, and post capture analysis of individual cells. The following papers present such microchip designs: Cho et al. designed a multilevel microfluidic platform for hydrogel scaffold construction in microchannels. The platform is constructed via a rapid prototyping method based on soft lithography using multilayered adhesive tape. The hydrogel scaffold is designed for 3D multicellular co-culturing and anticancer drug assays (tamixufen). Here, co-culturing of breast cancer cells (MDA-MB-231) and fibroblasts (NIH/3T3) in separate compartments was demonstrated with $>98 \%$ viability. Iqbal et al. designed a tapered channel device for studying the inhibition and migration of human glioblastoma (hGBM) cells when they were exposed to a novel aptamer targeting epidermal growth factor receptors (EGFR). Integration of this system with a microscope provides real-time and quantitative characterization of tumor cell behavior in vitro. This example demonstrates the capability of a microchannel device for integrated cell culturing and drug assay. White et al. designed a novel microdevice that enables integration of the mammosphere technique into a microsystem, thus combining it with a number of capabilities, such as cell sorting, drug screening, and molecular assays. In this paper, a poly(dimethyl siloxane) (PDMS) microdevice was utilized to effectively attach and promote the formation of mammospheres. Furthermore, reagents were exchanged within the mammosphere culture region by using a microporous membrane. Mammospheres were shown to be successfully cultured for ELISA testing. Li et al. designed a whole cell impedance-based biosensor to monitor the effects of a range of different frequencies at low-intensity on the growth rate of SKOV3 ovarian cancer cells and non-cancerous HUVECs. It was found that the growth profile of SKOV3 cells exhibited a significant decrease with application of $200 \mathrm{kHz}$ alternating electric fields whereas HUVEC cells exhibited little to no change in growth. This study serves to present data on inhibitory effects on dividing cancer cells in culture in contrast to non-cancerous cells. Such data can eventually be used in development of methods and optimal parameters for alternating electric field therapies in biomedical applications.

An increasingly important area of interest is to design and optimize microfluidic microchips for cancer cell isolation. In this special issue, Wang et al. designed and modeled magneto-nanosensors made of arrays of GMP spin-valves for enhanced detection of representative biomarkers, i.e. phosphor-SMC1, GCSF, and IL-6, for radiation exposure and cancer. The design utilized a new analytical resistive network model to optimize a previous device design. Realtime signals generated in the form of resistance changes of the spin-valve sensors were induced by a magnetic field from superparamagnetic nanoparticles. The new design resulted in enhanced sensitivity of two to three orders of magnitude in the detection of biomarkers. Zhang et al. designed a microfluidic chip for the detection of CTCs in clinical blood samples. The microchip utilized an immunoagnetic-based approach for capturing CTCs. It was demonstrated that the direction of RBC sedimentation relative to magnetic force required for cell separation was important for capture efficiency, throughput, and purity. A novel flip-flop operation reduced stagnation of RBCs and non-specific binding on the capture surface by alternating the direction of magnetic field with respect to gravity. High capture rates (more than $90 \%)$ were achieved. In addition, the magnetic field distribution was optimized with a smoothened magnetic gradient within the microchannel for more uniform distribution of particles on CTC capture plane. Alternatively, Luo and Qi et al. developed a cell self-loading and patterning device for quantitatively study the effect of density on cell behaviors. Using such model system, they correlate the local autocrine concentration with the tumor proliferation.

There is also an increasing interest in point-of-care detection systems. Much effort has been directed towards the development of inexpensive, disposable biosensor platforms using chemical surface modifications for wide range of biomedical applications. For example, Jon et al. designed nanobeads of cyclic olefin copolymer (COC) plastic surfaces for multilayerbased immunoassays. The surface of the nanobeads was chemically modified to enhance specificity in the detection of biomolecules. Amphiphilic copolymers of hydrophobic dodecyl or benzyl moiety, poly(ethylene glycol) (PEG), and an activated 
ester or epoxide functional group was used to coat COC substrates. Assembled into multilayers, the nanobeads achieved a dynamic range of detection $(0.1 \mathrm{ng} / \mathrm{mL}$ to $1,000 \mathrm{ng} / \mathrm{mL})$ and enhanced detection sensitivity $(0.1 \mathrm{ng} / \mathrm{mL})$ versus conventional detection sensitivity $(10 \mathrm{ng} / \mathrm{mL})$.

Recently, groups have studied other methods to address the issues surrounding conventional CTC isolation methods, such as reliance on the size and immunoaffinity of the cancer cells. The following papers suggest other methods that overcome these issues: Gourkutty Sanjay et al. demonstrate a negative enrichment method which integrates WBC depletion and chemical-free RBC depletion within the same setup. The method was designed to accomplish tumor cell isolation from blood samples with minimal sample handling and minimal tumor cell loss without relying on cell morphology or surface biomarkers for capture. It thus addressed two major issues of contemporary CTC isolation techniques. WBCs were first depleted by immunomagnetic assay, followed by RBC depletion via microfiltration. As a result, the method achieved $>90 \%$ recovery of spiked tumor cells. In order to investigate the potential of nanoscale technologies for targeted cancer drug delivery, Ramasamy et al. developed a nano-based drug delivery system. In this platform the nanoparticles were prepared from PEG surface modified hydroxyapatite and titanium dioxide nanoparticles. For tumor targeting, they functionalized the generated nanoparticles with folic acid molecules. In vitro and in vivo results show significant enhancement of paclitaxel drug delivery efficiency in the treatment group with targeted nano delivery system compared to the control groups. This work illustrates the potential of nanoscale technologies for improved cancer drug therapy.

To summarize, in this special issue we present a series of recent papers that address the current status and fundamental issues related to the development of multi-scale tumor models using microfluidics and nanoscale devices, and explore novel methods on how we can harness the power of micro- and nanotechnologies to significantly change the way we diagnose and treat cancer.

Acknowledgments We would like to express our sincere appreciation to all the authors for their contribution to this special issue. We also thank the reviewers for their time and invaluable comments. We gratefully acknowledge the financial support from the National Institute of Health (NIH) under the grant 1R01CA139070. 